\title{
Brief Original Article \\ Presence of different beta-lactamase classes among clinical isolates of Pseudomonas aeruginosa expressing AmpC beta-lactamase enzyme
}

\author{
Supriya Upadhyay ${ }^{1}$, Malay Ranjan Sen ${ }^{1}$, Amitabha Bhattacharjee ${ }^{2}$ \\ ${ }^{1}$ Department of Microbiology, Institute of Medical Sciences, Banaras Hindu University, Varanasi-221005, India \\ ${ }^{2}$ Department of Microbiology, Assam University, Silchar-788011, India
}

\begin{abstract}
Introduction: Infections caused by Pseudomonas aeruginosa are difficult to treat as the majority of isolates exhibit varying degrees of betalactamase mediated resistance to most of the beta-lactam antibiotics. It is also not unusual to find a single isolate that expresses multiple $\beta$ lactamase enzymes, further complicating the treatment options. Thus the present study was designed to investigate the coexistence of different beta-lactamase enzymes in clinical isolates of $P$. aeruginosa.

Methodology: A total of 202 clinical isolates of P. aeruginosa were tested for the presence of AmpC beta-lactamase, extended spectrum betalactamase (ESBL) and metallo beta-lactamase (MBL) enzyme. Detection of AmpC beta-lactamase was performed by disk antagonism test and a modified three-dimensional method, whereas detection of ESBL was done by the combined disk diffusion method per Clinical and Laboratory Standards Institute (CLSI) guidelines and MBL were detected by the Imipenem EDTA disk potentiation test.

Results: A total of $120(59.4 \%)$ isolates were confirmed to be positive for AmpC beta-lactamase. Among them, 14 strains (7\%) were inducible AmpC producers. Co-production of AmpC along with extended spectrum beta-lactamase and metallo beta-lactamase was reported in $3.3 \%$ and $46.6 \%$ isolates respectively.

Conclusion: The study emphasizes the high prevalence of multidrug resistant $P$. aeruginosa producing beta-lactamase enzymes of diverse mechanisms. Thus proper antibiotic policy and measures to restrict the indiscriminative use of cephalosporins and carbapenems should be taken to minimize the emergence of this multiple beta-lactamase producing pathogens.
\end{abstract}

J Infect Dev Ctries 2010; 4(4):239-242.

(Received 10 September 2009 - Accepted 7 December 2009)

Copyright () 2010 Upadhyay et al. This is an open-access article distributed under the Creative Commons Attribution License, which permits unrestricted use, distribution, and reproduction in any medium, provided the original work is properly cited.

\section{Introduction}

Infections caused by Pseudomonas aeruginosa are difficult to treat as the majority of isolates exhibit varying degrees of innate resistance. Acquired resistance is also reported by the production of plasmid mediated AmpC beta ( $\beta$ )-lactamase, extended spectrum $\beta$-lactamase and metallo $\beta$ lactamase (MBL) enzymes [1]. With the increase in occurrence and types of these multiple $\beta$-lactamase enzymes, early detection is crucial, the benefits of which include implementation of proper antibiotic therapy and infection control policy. At present Clinical and Laboratory Standards Institute (CLSI) guidelines do not describe any method for detection of these enzymes in $P$. aeruginosa. Hence the present study was designed to investigate the presence of different classes of $\beta$-lactamase enzymes in clinical isolates of $P$. aeruginosa with special reference to inducible and non-inducible AmpC $\beta$-lactamases.

The study included a total of 202 consecutive nonduplicate isolates of $P$. aeruginosa obtained from different clinical specimens from patients who were admitted in different wards $(n=159)$ as well as from those who attended the outpatient departments $(\mathrm{n}=$ 43) of our tertiary referral hospital. The study was conducted for six months from March 2008 to September 2008.

Screening for AmpC $\beta$-lactamase production was performed by Cefoxitin disk test. Isolates that yielded a zone diameter less than $18 \mathrm{~mm}$ (screen positive) were further subjected to confirmatory testing.

The disk antagonism test was used for detection of inducible AmpC $\beta$-lactamase in all the isolates of $P$. aeruginosa. A test isolate (with a turbidity equivalent to that of $0.5 \mathrm{McF}$ arland standards) was spread over a Mueller Hinton agar (Hi-Media) plate. Cefotaxime $(30 \mu \mathrm{g})$ and cefoxitin $(30 \mu \mathrm{g})$ (Hi-Media Mumbai) disks were placed $20 \mathrm{~mm}$ apart from centre to centre. Isolates showing blunting of the cefotaxime zone of inhibition adjacent to the cefoxitin disk were screened as positive for AmpC $\beta$-lactamase (Figure. 1A). Further confirmation of AmpC production was tested by a modified three-dimensional test as described previously [1]. This method was particularly helpful in detecting non inducible AmpC B-lactamases (Figure 1B). 
Figure 1 (A). Isolates showing blunting of the cefotaxime zone of inhibition adjacent to cefoxitin disk are AmpC beta lactamase producers; (B) Organism showing clear distortion in the zone of inhibition strain A (test strain), B (positive control) and minimal distortion (strainC) are AmpC producers, and no distortion strain D (negative control) indicates nonAmpC producers; (C) Isolate showing ESBL production, zone of inhibition given by the Ceftazidime+ clavulanic acid disk is $\geq 5 \mathrm{~mm}$ than those of Ceftazidime disk alone; (D) MBL production, Zone of inhibition given by the Imipenem+EDTA disk is $\geq 7 \mathrm{~mm}$ than those of Imipenem disk alone.

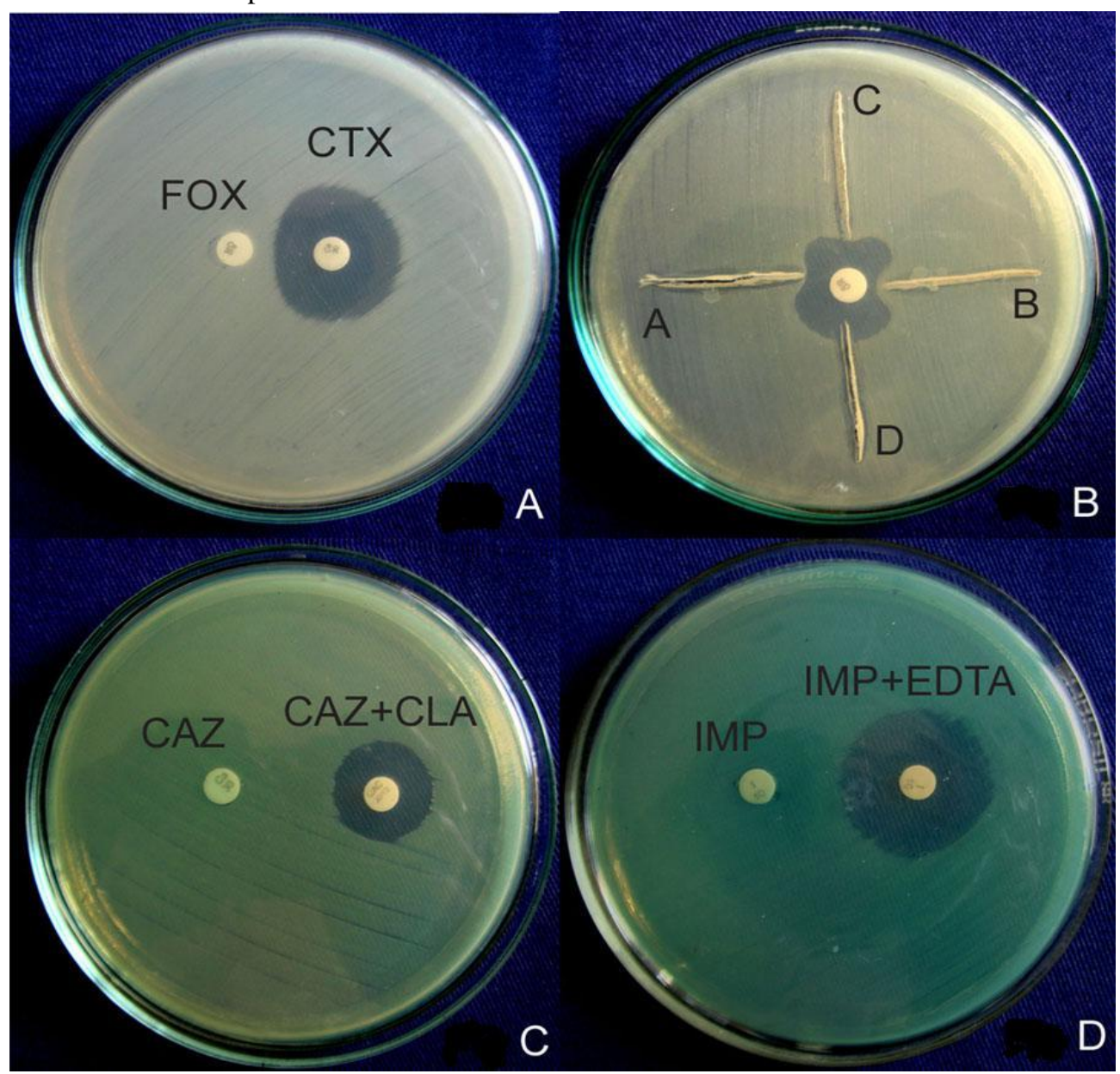

FOX- Cefoxitin; CTX- Cefotaxime; CAZ- Ceftazidime; CAZ+CLA- Ceftazidime + Clavulanic acid; IMP- Imipenem; IMP+EDTA- Imipenem + EDTA.

The extended spectrum beta-lactamase( ESBL) status of these strains was established by combined disk diffusion method per CLSI recommendations [2] using Cefotaxime $(30 \mu \mathrm{g})$ and Ceftazidime $(30 \mu \mathrm{g})$ disks alone and in combination with clavulanic acid (Figure 1C). Metallo $\beta$-lactamase production was detected by Imipenem-EDTA disk test. Two $10 \mu \mathrm{g}$ imipenem disks were placed on the plate, and appropriate amounts of $10 \mu \mathrm{l}$ of $0.5 \mathrm{M}$ EDTA solution were added to one of them to obtain the described concentration $(750 \mu \mathrm{g})$. The inhibition zones of imipenem and imipenem-EDTA disks were compared after 16 to 18 hours of incubation in air at $35^{\circ} \mathrm{C}$. If the increase in inhibition zone with imipenem and EDTA disk was $\geq 7 \mathrm{~mm}$, then the imipenem disk alone was considered to be the MBL producer [3] (Figure 1D).

Antimicrobial susceptibility was performed by the Kirby-Baeur disk diffusion method for various antibiotics, namely: Ampicillin $(30 \mu \mathrm{g})$, Amikacin $(30 \mu \mathrm{g})$, Co-trimoxazole $(25 \mu \mathrm{g})$, Ciprofloxacin $(5 \mu \mathrm{g})$, Ceftizoxime $(30 \mu \mathrm{g})$, Cefuroxime $(30 \mu \mathrm{g})$, Kanamycin $(30 \mu \mathrm{g})$, Piperacillin $(100 \mu \mathrm{g})$, Netilmicin $(30 \mu \mathrm{g})$, Gentamicin $(10 \mu \mathrm{g})$, Piperacillin/ tazobactam $(100 \mu \mathrm{g} / 10 \mu \mathrm{g})$, Carbenicillin $(100 \mu \mathrm{g})(\mathrm{Hi}-\mathrm{Media}$ 
Table 1. Total number of inducible as well as non-inducible AmpC $\beta$-lactamase producers.

\begin{tabular}{|c|c|c|c|}
\hline \multirow{2}{*}{$\begin{array}{c}\text { Total } \\
\text { Number of } \\
\text { isolates }\end{array}$} & Screening Positive & \multicolumn{2}{|c|}{ Confirmatory positive } \\
\cline { 2 - 4 } & & $\begin{array}{c}\text { Disc antagonism test } \\
\text { (inducible AmpC producers) }\end{array}$ & Modified three dimensional test \\
(Inducible and non-inducible AmpC producers)
\end{tabular}

Table 2. Different $\beta$-lactamase mediated resistance mechanism in AmpC producing Pseudomonas aeruginosa $(\mathrm{n}=120)$.

\begin{tabular}{|c|c|c|}
\hline AmpC & AmpC +ESBL & AmpC +MBL \\
\hline $60(50 \%)$ & $4(3.3 \%)$ & $56(46.6 \%)$ \\
\hline
\end{tabular}

Table 3. Antibiotic susceptibility pattern of P. aeruginosa.

\begin{tabular}{cc}
\hline Antimicrobial agents & $\%$ susceptibility \\
\hline Ampicillin & $7(3.4 .0 \%)$ \\
Amikacin & $96(47.5 \%)$ \\
Carbenicillin & $88(43.5 \%)$ \\
Cefoxitin & $6(3.0 \%)$ \\
Ceftazidime & $35(17.3 \%)$ \\
Ceftizoxime & $22(10.8 \%)$ \\
Cefuroxime & $5(2.4 \%)$ \\
Co-trimoxazole & $88(43.5 \%)$ \\
Ciprofloxacin & $64(31.7 \%)$ \\
Gentamicin & $79(39.1 \%)$ \\
Imipenem & $180(89.1 \%)$ \\
Netilmicin & $83(41.1 \%)$ \\
Piperacillin & $72(35.6 \%)$ \\
Piperacillin + Tazobactam & $102(50.5 \%)$ \\
Kanamycin & $14(7.0 \%)$ \\
\hline
\end{tabular}

Mumbai). The results were interpreted according to the standard table provided along with the disk.

Out of $202 P$. aeruginosa isolates tested, cefoxitin resistance was evident in $196(97 \%)$ isolates while $120(59.4 \%)$ isolates were confirmed to be AmpC $\beta$-lactamase producers. Among the test isolates, $7 \%(14 / 202)$ were detected as inducible AmpC producers while 52.4\% (106/202) of the isolates were confirmed to be non-inducible. The coexistence of AmpC and ESBL was reported in 4/120 (3.3\%) isolates, whereas AmpC and co-production of MBL was shown by $46.6 \%(56 / 120)$ of the isolates. Among these $56 \mathrm{MBL}$ producers, 23 isolates were found to show resistance towards Imipenem.
All the AmpC $\beta$-lactamase producing $P$. aeruginosa were multidrug resistant. Maximum sensitivity $(89.1 \%)$ was seen with imipenem, followed by moderate activity with piperacillin/tazobactam (51.5\%), amikacin (47.5\%), carbenicillin (43.5\%) and co-trimoxazole (43.5\%), and poor susceptibility patterns with the remainder of the drugs.

Production of multiple $\beta$-lactamases by $P$. aeruginosa has tremendous therapeutic consequences and poses a significant clinical challenge if it remains undetected. Since these organisms also carry other drug-resistant genes and the only viable treatment option remains the potentially toxic polymyxin B and colistin [4], early identification of the infections due 
to these organisms is necessary as the appropriate treatment might reduce the spread of these resistant strains as well as reduce the mortality in hospitalized patients. This emphasizes the need for the detection of isolates that produce these enzymes to avoid therapeutic failures and nosocomial outbreaks. Since there is no standard guideline for detection of most of these $\beta$-lactamase enzymes in $P$. aeruginosa, the comparison between studies becomes difficult as the patient population in particular centres and the methods of study differ. In comparison to the earlier studies conducted at other centres, we found a very high prevalence of AmpC $\beta$-lactamase producing $P$. aeruginosa (59.4\%) [5]. The increase in AmpC producing isolates may be indicative of the ominous trend of more and more isolates acquiring resistance mechanisms rendering the antimicrobial armamarium ineffective. Our study reported very low incidence of ESBL among $P$. aeruginosa $(3.3 \%)$, which contrasts an earlier study which showed $20.27 \%$ of ESBL production [6]. The only $\beta$-lactam active against coAmpC and ESBL producers are carbapenems; however, recently resistance to carbapenems has been increasing, which is mostly due to the production of MBL [4]. Carbapenem hydrolysing MBLs have been reported in several countries and have emerged as the most important mechanism of carbapenem resistance $[7,8]$. Our findings showed a high percentage of MBL producing $P$. aeruginosa $(46.6 \%)$ among AmpC producing isolates; however, earlier studies in this country showed low $(7.5 \%)$ to moderate $(20.8 \%)$ prevalence of MBLs $[9,10]$. In our study imipenem was found to be the most effective drug, showing maximum susceptibility of $89.1 \%$, which is in agreement with earlier studies $[6,11]$.

The present study emphasizes the high prevalence of multidrug resistant Pseudomonas aeruginosa producing $\beta$-lactamase enzymes of diverse mechanisms. To combat these problems, epidemiological studies should be undertaken in hospital settings to monitor the source of infection. Early detection of these $\beta$-lactamase producing isolates in a routine laboratory could help to avoid treatment failure, as often the isolates producing this enzyme show a susceptible phenotype in routine susceptibility testing. Furthermore, strict antibiotic policies and measures to limit the indiscriminative use of cephalosporins and carbapenems in the hospital environment should be undertaken to minimize the emergence of this multiple $\beta$-lactamase producing pathogen whose spread would leave no other option to treat Gram-negative nosocomial infections.

\section{Acknowledgements}

We would like to acknowledge the Head of the Department of Microbiology, Institute of Medical Sciences, Banaras Hindu University, Varanasi, for providing financial support to conduct the study.

\section{References}

1. Manchanda V, Singh NP (2008) Occurrence and detection of AmpC b-lactamases among Gram negative clinical isolates using a modified three-dimensional test at Guru Tegh Bahadur Hospital, Delhi, India. J Antimicrob Chemother 51: 415-418.

2. CLSI: Perfomance Standards for antimicrobial disc susceptibility tests. CLSI:Wayne PA; 2005-p. M100-S15.

3. Yong D, Lee K, Yum JH, Shin HB, Rossolini GM, Chong Y (2002) Imipenem-EDTA disk method for differentiation of metallo $\beta$ lactamase-producing clinical isolates of Pseudomonas spp. and Acinetobacter spp. J Clin Microbiol 40: 3798-801.

4. Livermore DM, Woodford N: Carbapenemase (2000) A problem in waiting? Curr Opin Microbiol 3:489-95.

5. Arora S and Bal M (2005) AmpC $\beta$-lactamase producing bacterial isolates from kolkata hospital. Indian J Med Res 122: 224-233.

6. Aggarwal R, Chaudhary U, Bala K (2008) Detection of extended spectrum beta lactamase in Pseudomonas aeruginosa. Indian J Patho Microbiol 51: 222-224.

7. Hancock RE (1998) Resistance mechanisms in Pseudomonas aeruginosa and other non fermentative gram negative bacteria. Clin Infect Dis 27: 593-599.

8. Nordman P, Poirel L (2002) Emerging Carbapenemases in Gram negative aerobes. Clin Microbial Infect 8: 321-331.

9. Gupta V, Datta P, Chander J (2006) Prevalence of metallo beta lactamase (MBL) producing Pseudomonas spp. and Acinetobacter spp. in a tertiary care hospital in India. $\mathbf{J}$ Infect 52: 311-314.

10. Varaiya A, Kulkarni N, Kulkarni M, Bhalekar P, Dogra J (2008) Incidence of metallo beta lactamase producing Pseudomonas aeruginosa in ICU patients. Indian J Med Res 127: 398-402.

11. Hemlatha V, Sekar U, Kamat V (2005) Prevalence of metallo beta lactamase producing Pseudomonas aeruginosa in hospitalized patients. Indian J Med Res 122: 148-152.

\section{Corresponding author}

Malay Ranjan Sen

Department of Microbiology

Institute of Medical Sciences

Banaras Hindu University

Varanasi - 221005, India

Telephone: +919415820675

Email: mr_senbhu@yahoo.com

Conflict of interest: No conflict of interest is declared. 